\title{
DENDRITIC GROWTH OF ICE CRYSTALS
}

\author{
N. H. FLETCHER
}

Department of Physics, University of New' England, Armidale, N.S.W. 2351, Australia

Received 31 March 1973; revised manuscript received 7 August 1973

The dendritic branching of ice crystals grown from the vapour at temperatures between -10 and -20 " $\mathrm{C}$ shows some features which are not adequately described by the existing theory. It is proposed that surface diffusion driven by the gradient in concentration of surface adsorbed molecules may be an important mechanism for growth stabilization in ice and that growth to the form of a sector-plate occurs when, because of non-linearity in the adsorption isotherm, this stabilization is no longer adequate. It is further proposed that true dendritic growth may, in this case, be associated with a change in interface kinetics at high supersaturation.

\section{Introduction}

The habits of snow-flakes and of ice crystals grown from the vapour under laboratory conditions exhibit a variety and extremity of form ${ }^{1.2}$ ) and a symmetry of pattern which has long excited artistic and scientific curiosity and demanded explanation. Nakaya's original classification of growth morphology in terms of the temperature and supersaturation of the environment ${ }^{2}$ ) has been refined and extended by Hallett and Mason ${ }^{3}$ ) and by Kobayashi ${ }^{4,5}$ ) so that it is now clear that there are two almost independent influences: temperature and excess vapour density relative to ice. This modified Nakaya diagram, as developed by Kobayashi, is shown in fig. 1 .

Only for very low growth rates and excess vapour density less than about $0.04 \mathrm{~g} \mathrm{~m}^{-3}$ is the crystal morphology that which would be expected from Wulff's theorem, the shapes being nearly equiaxial hexagonal prisms. For faster growth rates the habit shows extreme variations, from needles with axial ratio greater than 10:1 to plates with axial ratio less than $1: 10$ over only a

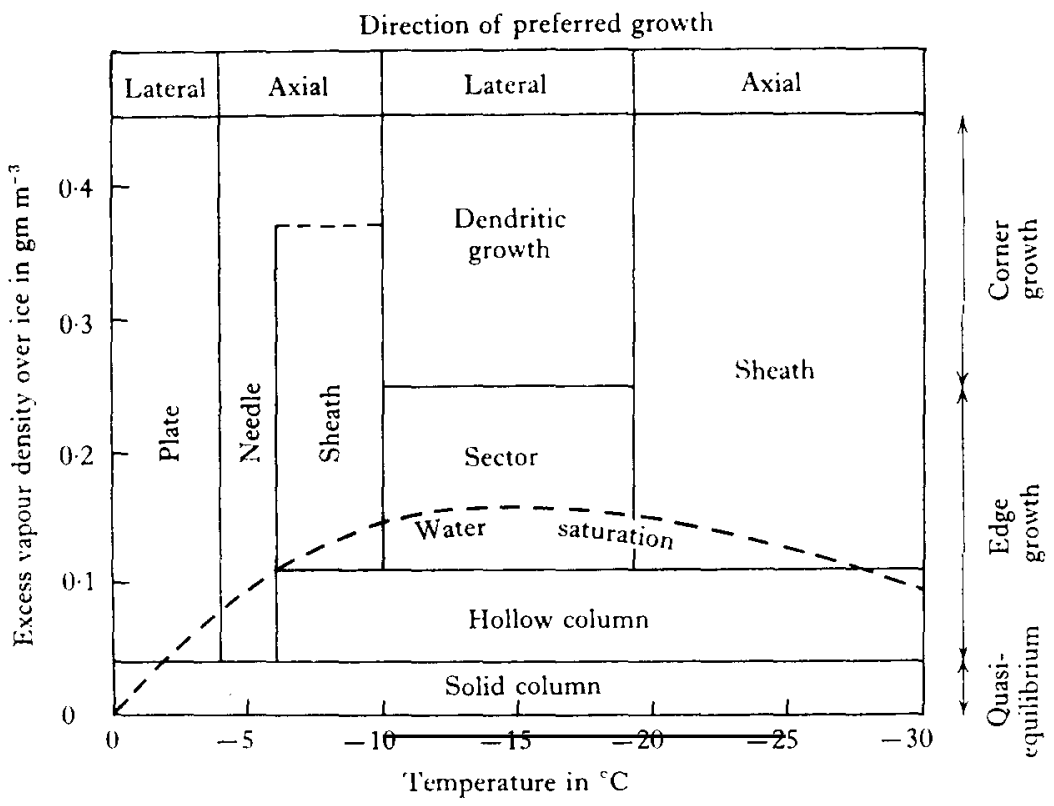

Fig. 1. The Nakaya diagram as modified by Kobayashi ${ }^{5}$ ). 
degree or so. Such variations can only be due to kinetic effects. The spectacular dendritic growth characteristic of snow-flakes takes place only at temperatures between about -10 and $-20{ }^{\circ} \mathrm{C}$ and for vapour density excess greater than about $0.25 \mathrm{~g} \mathrm{~m}^{-3}$. For excess vapour density in the range 0.1 to $0.25 \mathrm{~g} \mathrm{~m}^{-3}$ the morphology is usually described as a "sector plate" and consists of six individual hexagonal plate-like growths linked symmetrically to a central hexagon. Examples of both dendritic and sector crystals are given in plate 1 of ref. 6 as well as in refs. 1 and 2 . Details of the growth morphology depend critically upon supersaturation but little upon crystal size.

Work over the past decade is leading to an understanding of dendritic growth in general and there is now a general concensus on the origin of the extreme habit changes with temperature in ice. There remain, however, several important aspects of the ice dendrite problem which are not implicit in present theoretical treatments. It is the purpose of the present paper to propose an explanation for these.

\section{Surface diffusion and habit change}

Because the extreme habit variations in ice crystals must be kinetic and have been observed only in the ice system, their explanation must be sought in a kinetic mechanism at the ice/vapour interface with a strong temperature variation peculiar to ice. The surface phase change a few degrees below the melting point, proposed long ago by Faraday and given more detailed treatment by the present author ${ }^{7}$ ) provides such a possibility, although its reality has yet to be conclusively demonstrated. Hallett ${ }^{8}$ ) and Mason et al. ${ }^{9}$ ) have found a strong oscillatory behaviour of the surface diffusion length on the basal surface of ice in the range -10 to $0{ }^{\circ} \mathrm{C}$ which could be the result of this phase change and could supply the necessary kinetic mechanism for habit change ${ }^{9}$ ). There are some remaining difficulties in correlating the direction of change in diffusion length with the resulting crystal habit, but these will not concern us here.

\section{Dendritic growth}

Present understanding of the morphological instability process leading to dendritic growth is based upon the work of Mullins and Sekerka ${ }^{10}$ ). They showed that perturbations of a sphere growing in a diffusion field are unstable except for the modifying influence of surface free energy which stabilizes the shape for spheres of radius less than about seven times the critical radius for growth. This predicts, in a general way, dendritic growth at $10 \%$ supersaturation for spheres larger than a few tenths of a micron.

This theory has since been extended in various ways ${ }^{1-14}$ ). Coriell and Parker ${ }^{11}$ ), in particular, have shown that surface diffusion provides a further stabilizing mechanisms by tending to destroy any perturbations on the growth surface. The stabilization effect of surface diffusion is very considerable and might typically lead to stability up to radii of nearly $1 \mu \mathrm{m}$ for a $10 \%$ supersaturation.

If ice has an anomalously high surface diffusion coefficient, which is possible, then this theory may be able to account for the persistent growth of ice crystals as featureless hexagonal plates up to diameters of 50 $\mu \mathrm{m}$ or more at low vapour density excess. Because the surface diffusion in the theory is driven by the thermodynamic force of surface curvature, it is independent of vapour density excess in the environment so that its stabilizing influence is less at greater supersaturations. This is in agreement with the greater tendency toward dendritic growth at large supersaturations. While subsequent papers ${ }^{12,13}$ ) have included the effects of interface kinetics in the development, none seems to have taken account of differential surface adsorption as an additional driving mechanism for surface diffusion.

Now the situation with which we are concerned is one of large supersaturation in the environmenc, and direct experiments ${ }^{6,7}$ ) have shown that even under these conditions ice surfaces can support the associated vapour density excess without growth other than by step migration. Under these circumstances, however, the density of surface adsorbed molecules must be appreciable and must depend upon the vapour density excess at the surface. Since this quantity varies largely from one part of the crystal to another, the gradient of concentration of adsorbed molecules must provide a further stabilizing process against dendrite formation.

\section{Surface diffusion and dendritic growth}

To make our point about the importance of this mechanism, it is not necesary to solve the crystal growth problem completely; a semi-quantitative solution will be adequate. Consider then the growth of a hexagonal 
plate crystal and suppose that the growth mechanism on basal faces is so slow that they are effectively inert. Let us concentrate attention on one of the crystal prism faces of length $2 /$ and thickness $t$ and divide it into regions $A$ and $B$ as shown in fig. 2. Suppose that the average vapour density at the $\mathrm{A}$ surface is $\rho_{\mathrm{A}}$ and that at the B surface $\rho_{B}$, while the vapour density at a large distance is $\rho_{\%}$.

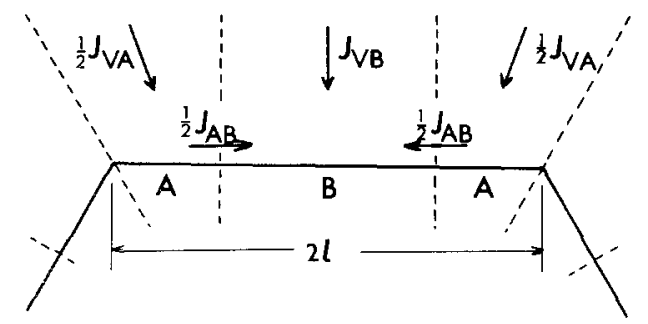

Fig. 2. Growth regions and fluxes for a hexagonal plate, as defined in the text.

The rate of vapour deposition onto the two surfaces A can be drived from dimensional analysis and involves the product $l^{x} t^{y}$ where $x+y=1$. From fairly general considerations we expect $x \simeq y$, though the detailed relation between them may depend on the angle of the crystal vertex and the ratio $l / t$. It is therefore a reasonable approximation to write

$J_{\mathrm{VA}}=\alpha D_{\mathrm{V}}\left(\rho_{\alpha}-\rho_{\mathrm{A}}\right)(t t)^{\frac{1}{2}}$,

where $D_{\mathrm{v}}$ is the vapour diffusion coefficient and $\alpha$ is a numerical coefficient of order unity, determined solely by the geometry of the crystal and independent of its size. Similarly the rate of deposition onto surface $B$ is

$J_{\mathrm{VB}}=\beta D_{\mathrm{V}}\left(\rho_{\gamma}-\rho_{\mathrm{B}}\right)(l t)^{\frac{1}{2}}$,

where $\beta$ is a similar constant. For a normal convex crystal $\alpha>\beta$.

Now for growth by a lateral mechanism in which steps are nucleated at the corners in region $A$ and then propagate across the surface through regions $A$ and $B$ in turn, the growth velocity is independent of the surface adsorbed concentrations $n_{\mathrm{A}}$ and $n_{\mathrm{B}}$ provided they are both greater than the equilibrium value, and depends only on the flux of molecules to the region concerned. This is so because the growth steps pile up in regions of low surface adsorbed concentration and so balance the growth rate.

The process tending to equalize these two fluxes is surface diffusion from $\mathrm{A}$ to $\mathrm{B}$ and, if we suppose $n_{\mathrm{A}}$ and $n_{\mathrm{B}}$ to be the surface adsorbed concentrations of molecules in regions $A$ and $B$ respectively, then we can write this diffusion flux as essentially

$J_{\mathrm{AB}}=D_{\mathrm{S}}\left(n_{\mathrm{A}}-n_{\mathrm{B}}\right) m t / l$

where $D_{\mathrm{S}}$ is the surface diffusion coefficient for adsorbed molecules and $m$ the molecular mass.

The condition for non-dendritic growth is thus simply $J_{\mathrm{A}}=J_{\mathrm{B}}$ or

$J_{\mathrm{VA}}-J_{\mathrm{VB}}=2 J_{\mathrm{AB}}$.

Using (1), (2) and (3) this becomes

$\alpha\left(\rho_{x}^{\prime}-\rho_{\mathrm{A}}^{\prime}\right)-\beta\left(\rho_{x}^{\prime}-\rho_{\mathrm{B}}^{\prime}\right)=2 m D_{\mathrm{S}} t^{\frac{1}{2}} l^{-\frac{1}{2}} D_{\mathrm{V}}^{-1}\left(n_{\mathrm{A}}^{\prime}-n_{\mathrm{B}}^{\prime}\right)$,

where the primes on the quantities $\rho_{i}^{\prime}$ and $n_{i}^{\prime}$ imply the deviation of these quantities from their equilibrium values under exactly saturated conditions.

It is clear that (5) is satisfied identically for $\rho_{x}^{\prime}=0$ and that since $\rho_{\mathrm{A}}^{\prime}, \rho_{\mathrm{B}}^{\prime} \ll \rho_{x}^{\prime}$ it is possible to satisfy it also for small values of $\rho_{x}^{\prime}$, provided $/$ is sufficiently small. We need not be concerned with the details of such a solution, which depend upon the exact relation between the adsorbed concentration $n_{i}^{\prime}$ and vapour density $\rho_{i}^{\prime}$ above the surface. If such a solution exists for a given $/$ and $t$ however, and the $n_{i}^{\prime}$ are proportional to the related $\rho_{i}^{\prime}$ (as is the case for a simple adsorption isotherm at low coverage), then a linearily scaled solution also exists for any higher $\rho_{\alpha}^{\prime}$ for the same $l$ and $t$.

The relation between $n_{i}^{\prime}$ and $\rho_{i}^{\prime}$ is, however, not linear as $\rho_{i}^{\prime}$ increases. Rather, $n_{i}^{\prime}$ tends to saturate at a coverage near a monolayer (or perhaps in a rather more complicated way). When this occurs, the relative increase of the right-hand side of (5) with increasing vapour pressure is less than that of the left side and the equality can no longer be satisfied. There is, it is true, a small margin of adjustment in $\rho_{\mathrm{A}}^{\prime}$ and $\rho_{\mathrm{B}}^{\prime}$ but, since they are much less than $\rho_{\alpha,}^{\prime}$, this is soon exhausted. Once (5) can no longer be satisfied, the nett flux to the A regions exceeds that to the $\mathrm{B}$ region and the corners begin to grow more rapidly than the central parts of the crystal edges.

In order-of-magnitude terms, if we put $n_{\mathrm{A}}^{\prime}=n_{1}$, the monolayer coverage, and approximate $n_{\mathrm{B}}^{\prime}=0$ and $\rho_{\mathrm{A}}^{\prime}, \rho_{\mathrm{B}}^{\prime} \ll \rho_{x_{c}}^{\prime}$, the criterion for dendritic growth then becomes

$\rho_{x}^{\prime}>2 D_{\mathrm{S}} n_{1} m t^{\frac{1}{2}} / D_{\mathrm{v}} l^{\frac{3}{2}}(\alpha-\beta)$. 
As pointed out before $\alpha$ and $\beta$ are simple numerical coefficients of order unity, determined by the geometry of the crystal with $\alpha>\beta$. It is clear from the dependence of $\rho_{\infty}^{\prime}$ on $l$ and $t$ that a large thin crystal will make the transition to dendritic growth at a smaller vapour density excess than will a small thick crystal.

To evaluate the implications of (6) for ice, we note that $D_{\mathrm{v}} \simeq 0.2 \mathrm{~cm}^{2} \mathrm{~s}^{-1}, n \simeq 10^{15} \mathrm{~cm}^{-2}, m \simeq 3 \times 10^{-23}$ $\mathrm{g}$ and a reasonable value for $(\alpha-\beta)$ for a hexagon is $\sim 0.3$. No reliable value for $D_{\mathrm{S}}$ appears to be available in the literature but Coriell and Parker ${ }^{13}$ ) take a typical value as $10^{-3} \mathrm{~cm}^{2} \mathrm{~s}^{-1}$.

Hobbs and $\operatorname{Scott}^{16}$ ) have re-examined the stepgrowth data of Hallett ${ }^{8}$ ) and of Mason et al. ${ }^{9}$ ) to derive a value of $1.3 \times 10^{-5} \mathrm{~cm}^{2} \mathrm{~s}^{-1}$ for $D_{\mathrm{s}}$ on basal faces of ice at $-10{ }^{\circ} \mathrm{C}$ but the uncertainties in their treatment are such that this estimate could well be in error by more than an order of magnitude and, in any case, $D_{\mathrm{S}}$ for prism faces is apparently much larger than $D_{\mathrm{S}}$ for basal faces in the temperature range of our present interest. We therefore adopt the value assumed by Coriell and Parker for an illustrative calculation.

For a crystal $100 \mu \mathrm{m}$ in diameter and $10 \mu \mathrm{m}$ thick we have $l=2.5 \times 10^{-3} \mathrm{~cm}, t=10^{-3} \mathrm{~cm}$ and (6) predicts dendritic growth when the vapour density excess exceeds $0.2 \mathrm{~g} \mathrm{~m}^{-3}$. The good agreement between this value and the observed threshold of $0.1 \mathrm{~g} \mathrm{~m}^{-3}$ is clearly fortuitous but the order of magnitude agreement is significant. It is clear that surface diffusion of adsorbed molecules is a very efficient stabilization mechanism for growth morphology, at least an order of magnitude better in this case than the mechanism proposed by Coriell and Parker.

\section{Sectorial and dendritic growth}

It is not possible from this semi-quantitative discussion to deduce any details of dendrite shape, but we can make some qualitative speculations. We note that the breakdown of stability is of a regional rather than a local nature so that the morphology should develop with a region of length about equal to the critical length $l^{*}$ at the prevailing supersaturation advancing fairly uniformly near each crystal corner. If a side is longer than $2 l^{*}$, a dip should develop near its midpoint. This is essentially the character of sector-plate growth.

True dendritic growth could be thought of as a limiting form of sectorial growth with very small $l^{*}$, but the experimental data suggest rather the sharp onset of a different growth regime. There seem to be two plausible mechanisms for this occurrence.

In the first place we note that, as the concentration of adsorbed molecules tends to uniformity at high vapour density excess, so the surface diffusion mechanism in this uniform region becomes inhibited and the stability problem reduces to the Mullins-Sekerka case. Locai instabilities may then arise in this particular region and give a different growth morphology.

Alternatively one might invoke the possibility of a different interface growth mechanism becoming active at high supersaturation. We have assumed a lateral mechanism but, for sufficient vapour supersaturation at the surface, a continuous advance with a rough interface or two-dimensional nucleation could become important. This mechanism would become active initially at the crystal corners where local supersaturation is highest and, by relieving this supersaturation, would effectively inhibit similar growth at neighbouring sites. A naive calculation of the required vapour density excess for such a mechanism yields a value in rough agreement with the observed dendritic threshold ${ }^{6}$ ).

This second mechanism of transition to true dendritic growth in ice crystals is in some ways similar to that proposed recently by Lacmann and Stranski ${ }^{16}$ ), although their mechanism was expressed in terms of the contact angle of an interfacial quasi-liquid film to which they ascribed macroscopic physical properties.

\section{Conclusion}

This semi-quantitative discussion has shown that surface diffusion of adsorbed molecules represents an important stabilizing influence on crystal growth. In the case of ice crystal growth, surface diffusion is postulated as being of major importance and it has been shown that this accounts for several features of the dendritic growth of snow-flakes.

The striking symmetry of snow-flakes is simply explained by the considerable anisotropy of the surface free energy and surface kinetics of ice, together with the nature of the dendritic growth criterion (6) which ensures that all six corners become dendritic essentially simultaneously. The dependence of this growth criterion on vapour density excess, together with the critical behaviour of the true dendrite threshold, allows sensi- 
tive response of crystal morphology to the vapour density in the environment.

\section{Acknowledgement}

This work was done in the course of a project supported by the Australian Research Grants Committee.

\section{References}

1) W. A. Bentley and W. J. Humphreys, Snow Crvstals (McGraw-Hill, New York, 1931).

2) U. Nakaya, Snow Crystals: Natural and Artificial (Harvard University Press, Cambridge, 1954).

3) J. Hallett and B. J. Mason, Proc. Roy. Soc. (London) A 247 ( 1958 ) 440.

4) T. Kobayashi, J. Met. Soc. Japan [2] 36 (1958) 193.

5) T. Kobayashi, Phil. Mag. 6 (1960) 1363.
6) N. H. Fletcher, The Chemical Physics of Ice (Cambridge University Press, Cambridge, 1970) ch. 5.

7) N. H. Fletcher, Phil. Mag. 7 (1962) 255; 8 (1963) 1425; 18 (1968) 1287.

8) J. Hallett, Phil. Mag. 6 (1960) 1073.

9) B. J. Mason, G. W. Bryant and A. P. van den Heuvel, Phil. Mag. 8 (1963) 505.

10) W. W. Mullins and R. F. Sekerka, J. Appl. Phys. 34 (1963) $323 ; 35$ (1964) 444.

11) S. R. Coriell and R. L. Parker, J. Appl. Phys. 37 (1966) 1548.

12) J. W. Cahn, in: Crystal Growth, Ed. H. S. Peiser (Pergamon. Oxford, 1967) p. 681.

13) S. R. Coriell and R. L. Parker, in: Crystal Growth, Ed. H. S. Peiser (Pergamon, Oxford, 1967) p. 703.

14) R. F. Sekerka, J. Crystal Growth 3/4 (1968) 71.

15) P. V. Hobbs and W. D. Scott, J. Geophys. Res. 70 (1965) 5025.

16) R. Lacmann and I. N. Stranski, J. Crystal Growth 13/14 (1972) 236. 